\title{
Pan-transcriptomic analysis of Solanum tuberosum NLR genes regulating resistance to Phytophthora infestans
}

\author{
Shmakov N.A. ${ }^{1,2,3 *}$, Kochetov A.V. ${ }^{1,2,3}$, Afonnikov D.A. ${ }^{1,2,3}$, Vasiliev G.V. ${ }^{1}$ \\ ${ }^{1}$ Institute of Cytology and Genetics, SB RAS, Novosibirsk, Russia \\ ${ }^{2}$ Kurchatov Genomic Center of the Institute of Cytology and Genetics, SB RAS, Novosibirsk, Russia \\ ${ }^{3}$ Novosibirsk State University, Novosibirsk, Russia \\ *email: shmakov@bionet.nsc.ru
}

Potato (Solanum tuberosum L.) is, according to FAOSTAT data, the sixth most important crop species in terms of production amounts. Potato yields can suffer from several biogenic and abiogenic factors. One of the most damaging biological factors is Phytophthora infestans, an oomycete pathogen that causes late blight of potato and tomato. A modern strategy of pathogen defense is identification of genetic markers of plant resistance to specific pathogen, which then can be used to create new strains, resistant to pathogen attacks. An important role in plant resistance to pathogens is played by proteins of NBS-LRR family, which identify pathogens and trigger immune response of the plant. In order to identify genetic markers of potato resistance to P. infestans, transcriptomes of four potato strains contrasting in susceptibility to the pathogen, and Solanum stoloniferum, a closely related wild member of Solanum genus, that is believed to be highly resistant to $P$. infestans, were studied. RNA-seq libraries of the plants were sequenced, and de novo transcriptome assembly was performed. After transcriptomes filtering and annotation, NBS-LRR domains were identified in the predicted protein sequences. Expression profiles of NBS-LRR genes were compared, and differential expression was observed for some of the NBS-LRR genes. Presence/absence variations and sequence variations between potato strains were observed for NBS-LRR genes. In addition, de novo assembled transcripts with no significant homology to genome were found, among which NBS-LRR genes were present. Thus, novel resistance genes of potato were identified. Finally, some of the known genes-markers of late blight resistance were identified in the assembled transcriptomes. Differences in sequences and expression profiles of marker genes were observed between potato strains.

Acknowledgements: This work was supported by the Kurchatov Genomic Center of the Institute of Cytology and Genetics, SB RAS (agreement No. 075-15-2019-1662). 